\title{
Déterminants Psycho-Socio-Culturels De La Victimisation Des Employées Mineures De Ménages À Abidjan
}

\author{
Par Nassoua Antoine Okpo \\ Maître-Assistant à l’UFR Criminologie, Université Félix Houphouët Boigny \\ de Cocody-Abidjan
}

doi: 10.19044/esj.2016.v13n2p211 URL:http://dx.doi.org/10.19044/esj.2016.v13n2p211

\begin{abstract}
This study aims to identify the psycho-sociocultural determinants of the victimization of minors employed by households. Two hypotheses have led to this research. Semi-directional interviews with parents and employers of minors were used. Three theories have been convened; The culturalist theory of the cultural transmission of values and beliefs, the theory of reasoned action and that of planned action have led to knowledge of the motivations of employers. The consciousness of the tacit guarantee of the impunity of the person who chastises a minor, the acceptance of punishments as a deterrent and educational force during socialization, and the subjective interpretation of the actions of minors are the main psychosociocultural determinants of the victimization of minors. These motivate and trigger the victim's attitude in the employer.
\end{abstract}

Keywords: Victimization, Subjective Interpretation, Corporal Punishment, Cultural Practices, Victimizer

\section{Résumé}

Cette étude vise à identifier les déterminants psycho-socioculturels de la victimisation des mineures employées de ménages. Deux hypothèses ont conduit cette recherche. Les entretiens semi-directifs avec les parents et employeurs de mineures ont été utilisés. Trois théories ont été convoquées ; la théorie culturaliste de la transmission culturelle des valeurs et croyances, la théorie de l'action raisonnée et celle de l'action planifiée ont abouti à la connaissance des motivations des employeurs. La conscience de la caution tacite quant à l'impunité de celui qui châtie une mineure, l'acceptation des châtiments comme forces dissuasive et éducative au cours de la socialisation, et l'interprétation subjective des actes des mineures sont les principaux 
déterminants psycho-socioculturels de la victimisation des mineures. Ceux-ci motivent et déclenchent chez l'employeur l'attitude victimelle.

Mots clés : Victimisation, Interprétation subjective, Châtiments corporels, Pratiques culturelles, Victimiseur

\section{Introduction}

Longtemps invisible, la victimisation des employées mineures de ménages est aujourd'hui fréquente, en témoignent les nombreuses mineures rencontrées à la brigade des mineurs, à la police, dans les centres sociaux et transférées au centre d'assistance éducative et de sauvetage du BICE du Plateau (Okpo, 2010). Cette tendance à victimiser les mineures provient d'un contexte sociologique des pratiques et représentations évoluant au cours des années. En effet, le travail des filles aidantes était, comme l'indique Jacquemin (2002), dans des logiques familiales, associées à un processus éducatif et de socialisation par le travail. Il faut comprendre par là que les femmes dans leurs différents foyers se faisaient aider par de jeunes filles à qui, elles inculquaient le savoir faire et les bonnes habitudes d'une femme au foyer. Cet apprentissage était vu comme une préparation à leurs futurs rôles et responsabilités d'une femme au foyer à l'âge adulte. Ces jeunes filles étaient heureuses d'apprendre au quotidien aux côtés de ces dames bienfaitrices. Jacquemin (2002) avance que ''la jeune parente, ou proche par l'origine régionale, la petite nièce n'est pas payée en numéraire : elle est logée, nourrie, habillée et assistée en cas de maladies par sa patronnetutrice qui devra également 'faire quelque chose' pour elle quand elle quittera la place. Une valise: trousseau, pagnes, vaisselle, machine à coudre, petit pécule, etc." composera les bagages qu'emportera la jeune fille. Lorsque ces filles étaient estimées prêtes, bien armées à affronter la vie conjugale, la bienfaitrice offrait des valises, des cantines pleines de vêtements, de pagnes auxquelles elle ajoutait des ustensiles de cuisine, tout le nécessaire du futur foyer de la jeune fille. Les parents de la jeune fille tout heureux comme leur enfant, bénissaient la bienfaitrice et cherchaient d'autres filles à lui confier. Faisant la fierté de sa famille, l'exemple de cette dernière sera suivi par d'autres filles qui ont volontairement choisi d'approcher les femmes des villes qui éprouvaient la nécessité d'avoir une jeune fille pour les aider dans leur foyer, sans toutefois tenir rigueur de l'origine régionale de la femme qui les accueille, de la patronne. Ces logiques sont aujourd'hui révolues. Nous sommes passés selon Jacquemin à des logiques davantage salariales. C'est pour ces raisons que cet apprentissage du travail féminin peut être envisagé comme le produit de caractéristiques propres au travail domestique et le recours à une main-d'œuvre juvénile pour effectuer les tâches domestiques est une spécificité du travail domestique et non plus 
comme une socialisation par le travail. Les femmes accueillantes ou patronnes sont de plus en plus exigeantes et l'on observe des conditions de vie et de travail insoutenables bien dénoncées dans les medias. Mejjati (2002) relève à ce sujet, les difficiles conditions de travail des enfants. L'auteur soutient que les conditions de travail des petites bonnes présentent de nombreuses caractéristiques des pires formes : précocité, responsabilités et tâches nombreuses, longueur des journées de travail, instabilité juridique, contraintes physiques et psychologiques constituent l'ensemble ou l'ossature des travaux qu'exercent les petites bonnes. Les motifs de mise au travail de ces filles sont très souvent la position socio-économique des parents. La pauvreté des ménages, leur faible niveau d'instruction et leur taille, c'est-àdire, le nombre de personnes au-delà de huit et atteignant souvent onze que comportent la plupart des ménages expliquent le travail précoce de ces filles. Il faut ajouter que cette raison fait comprendre pourquoi ces filles ne peuvent pas abandonner le travail domestique malgré la maltraitance subie.

Certains auteurs tels Chaoui, Gautier et Lnares ont insisté sur les facteurs explicatifs qui pérennisent la victimisation des employées domestiques. En effet, après la période où les parents confiaient leurs filles à des dames de la ville pour apprendre à leurs côtés et l'enfant devenue grande, rayonnante et prête pour la vie de son foyer rentrait les mains chargées pour une vie conjugale réussie, vient la période de la rémunération des filles aidantes. Se basant sur la somme à donner chaque fin de mois à ces filles qu'elles emploient, les employeures sont devenues très exigeantes avec ces dernières considérées comme des servantes, personnes à tout faire dans la maison et pourtant les femmes de ménage ne peuvent en réalité plus se passer d'elles. Cette exigence amène les femmes à mettre en place, plusieurs stratégies pour obtenir un travail bien fait, un rendement acceptable de la part des employées. Pour Echaoui (2009), ces filles et leurs parents considèrent le travail domestique chez un employeur comme une alternative à l'école et non comme une activité complémentaire. Ainsi, les filles interrogées citent très souvent, indique l'auteur, l'absentéisme des enseignants, la violence en classe, l'éloignement du collège, l'absence de sanitaires, une manière de justifier leur abandon de l'école et être libre psychologiquement, puisque la faute n’émane pas d'elles. Ces raisons sont parmi tant d'autres qui poussent les filles du monde rural à quitter l'école dès la fin du cycle primaire et à se refugier dans le travail quel qu'il soit. Aussi, ces petites filles sont prisonnières d'un système d'intermédiaires : les Samsara, bien que les seuls contacts de ces bonnes, ne peuvent les protéger des abus de leurs employeurs. L’auteur rapporte également que la pauvreté des parents et l'abandon scolaire en milieu rural alimentent le marché du travail domestique. La part de la culture est non moins négligeable. En effet, culturellement, beaucoup de familles favorisent des pratiques 
discriminatoires basées sur le sexe. Les filles se voient confier traditionnellement les tâches domestiques et le troisième facteur est l'existence d'un vide juridique; l'inexistence d'une loi réglementant le travail domestique. Tous ces facteurs réunis poussent consciemment ou inconsciemment les employeurs à victimiser les jeunes filles aidantes. Gautier (2004) bien que n’évoquant pas spécialement la victimisation des employées de ménages, expose les déterminants sociaux et institutionnels des violences au sein de la famille. La référence à Levinson l'amène à indiquer la détention des pouvoirs économique et décisionnel dans le ménage par le chef de ménage, la difficile décision pour la femme de divorcer, le recours des adultes à la violence pour résoudre leurs conflits et la répartition culturelle des rôles attribués à chaque sexe, notamment le fait que les hommes soient considérés comme les propriétaires de leur épouse sont autant de facteurs sociaux qui expliquent la violence dans la famille. Institutionnellement, l'Etat tolère dans la sphère privée, des actes qu'il reprouve dans la sphère publique ; le meurtre de la femme reste impuni si son adultère est prouvé comme au Paraguay ou en Haïti. Cette référence à Gautier montre la base culturaliste de la compréhension des victimisations dans les ménages. Il faut entendre par là, un déséquilibre dans les relations intrafamiliales. Le recours à Linares (2008) aide à mieux saisir le sens de ce déséquilibre relationnel. En effet, pour l'auteur, des abus et autres maltraitances sont dus à un certain dysfonctionnement des règles relationnelles. Il propose pour cela une vision et une stratégie thérapeutique qui peuvent apporter un véritable changement dans le champ relationnel. Ceci nous amène à projeter que ces femmes de ménages devraient plutôt chercher à profiter des services que leur rendent ces filles employées de ménages que de rendre la relation victimelle. La convocation de la culture pour la compréhension de la victimisation des employées mineures conduit à faire appel à certains auteurs qui ont relevé des pratiques sociétales ayant cours dans chacune.

Selon Gautier (2004), de nombreuses sociétés ont pratiqué ou pratiquent les châtiments corporels pour inculquer aux enfants les normes sociales en vigueur. La référence à Ojeda et al. conduit l'auteur à indiquer que certains peuples, comme en Colombie, jugent que le châtiment physique est nécessaire pour éduquer les enfants.

D’autres auteurs comme Moreno, Mejjati, et Okpo ont mis en évidence les formes des violences subies qui ne sont autres que les stratégies utilisées par les employeures pour obtenir des employées mineures, un rendement efficace, acceptable qui puisse mériter le salaire mensuel qu'elles gagnent. Ces stratagèmes employés pour rendre l'employée toujours prête à servir sont exposés par Mereno (2015). L’auteur décrivant la vie d'une petite ''bonne' au Maroc, expose les sévices de tout genre subis par la petite 
'bonne'. Ainsi, 'des coups, des frappes, des brûlures à la cigarette, des coups à la sandale ou à la babouche qui pleuvent sur la tête, le visage, les fesses, les pieds, le dos, le ventre sans pitié. Des privations de nourriture et des prises de repas seul dans un petit coin de cuisine et des séances de plaisir subies" représentent les sévices que subissent quotidiennement les petites bonnes. Mejjati (2002) abondait déjà dans ce sens en relevant les sévices physiques subis par les petites bonnes au Maroc. Ces petites sont exposées à des accidents, à des dermatoses et autres pour cause de manipulation d'outils tranchants et l'usage de détergents et de produits d'entretien souvent mal maîtrisés. Il s'agit également de mauvais traitements et des sévices physiques comme les bastonnades, les brûlures, les morsures, les pincées et les enfermements ainsi que des abus sexuels. Dans les ménages, Okpo (2010) indique également l'interprétation subjective des actes des enfants et adolescents justifiant leur victimisation. L'auteur expose les différentes formes de victimisations; violences physiques (sévices corporels, brûlures...), privation de nourritures, rejet affectif ; exclusion, isolement, maltraitance par omission, menaces et injures quotidiennes, abus sexuel, viol et attouchement sont les lots de violences subies par ces enfants et adolescents de la part des personnes qui en ont la charge. Il relève également quelques facteurs explicatifs tenant à une faute de la part de la victime et les jugements de valeur du victimiseur qui justifient les techniques de victimisation utilisées par les victimiseurs. Comme indiqué précédemment, ces stratégies proviendraient de la représentation individuelle et collective des stratégies éducatives pour inculquer les normes sociales aux mineurs.

Ainsi, ces stratégies sont usitées dans les familles à toutes les époques par les parents sur leurs propres enfants dans le but d'obtenir la valorisation, la révélation des capacités, des talents de ces enfants qui devront être les porte-flambeaux de leur famille. C'est ce que nous indique Raphaël (2015). En effet, le père de Céline lui inflige de mauvais traitements moraux (insultes, dévalorisation...) mais aussi physique (coups de ceinture, privation de nourriture...), va lui faire mener un parcours de combattant, un parcours inhumain, afin d'atteindre ses capacités musicales qu'il a décelées dès le plus jeune âge de sa fille. Salem (2010) avait déjà fait remarquer la maltraitance familiale en soulignant que $85 \%$ des enfants sont maltraités dans leur famille d'abord et non à l'extérieur. C'est surtout dans la famille proche que les enfants sont maltraités de nos jours, bien davantage que hors de la famille. L'auteur ajoute que les parents et la société entière sont clairement favorables à la fessée comme méthode éducative et pensent qu'une bonne correction, un bon châtiment physique peut faire du bien. Il nous parait alors important d'interroger la théorie culturaliste des valeurs et 
croyances culturelles pour comprendre cette attitude des parents envers leurs enfants au sein des familles.

C'est pour comprendre davantage et tenter d'apporter quelques éléments de réponse aux motivations des comportements des employeurs que la théorie culturaliste de la transmission culturelle des valeurs et croyances est associée à d’autres théories de l'action raisonnée et de l'action planifiée.

En effet, les théories s'appuyant sur les valeurs et croyances culturelles des différentes sociétés nous aident à mieux saisir le sens des violences dont sont victimes les enfants. Schoebi et al. (2006) soutiennent même que les différences entre cultures en ce qui concerne le recours à la punition corporelle comme moyen de socialisation ou comme moyen de contrôle dans l'interaction familiale ou sociale constituent un trait important pour caractériser les sociétés. Evoquant les facteurs facilitant la punition corporelle, ces auteurs avancent que beaucoup de facteurs sont susceptibles d'expliquer l'usage de punition corporelle dans certaines situations ou certaines sociétés. La référence à Dietz (2000), les amène à retenir finalement deux facteurs principaux tels le stress structurel, entendant par là, le bas statut socio-économique, les frustrations diverses et surtout une norme culturelle qui encourage l'utilisation de la force et de la violence comme une réponse aux frustrations. Ainsi, l’enfant ayant vécu cet environnement va intégrer ce mode d'agir lorsqu'il deviendra adulte. Coulibaly (2010) avance également des explications faisant référence aux valeurs et croyances culturelles en ce qui concerne les violences physiques directes pratiquées sur les enfants (élèves) par les parents (les enseignants). L'auteur recherchant les facteurs explicatifs de l'usage des châtiments corporels par les enseignants et les adultes dans les écoles africaines malgré leur interdiction officielle depuis les années 1970, exclut d'emblée le recours à la force publique donc une non pénalisation de ces pratiques, il indique plutôt les 'traditions' locales propices à de telles pratiques. Elles sont considérées comme plus puissantes que les lois et les règlementations. En effet, selon l'auteur, dans le système social global, les rôles et places des différentes catégories d'acteurs sont définis. Les adultes et les plus âgés disposent de fait, d'un droit de correction sur les enfants et les plus jeunes. Ainsi, les structures traditionnelles ont un poids déterminant dans l'organisation, le fonctionnement et les représentations sociales qui renforce et consolide le ciment social et culturel. Enseignants, parents et ainés se reconnaissent ainsi le droit d'user de la violence physique directe contre les élèves, les enfants et les cadets. Ce droit maintient les jeunes à leur "'place"' et ces stratégies sont jugées comme étant le meilleur moyen d'y parvenir. Elles se justifient dans la conscience collective des adultes et des jeunes par la nécessité de graver efficacement et durablement les savoirs, les valeurs et les compétences pour s’affranchir de 
son milieu. L’individu qui vit dans un tel milieu intègre consciemment ou inconsciemment ces pratiques.

La théorie de l'action raisonnée de Fishbein et Ajzen (Edu Tech Wiki, 2011) définit les liens entre les croyances, les attitudes, les normes, les intentions et les comportements des individus. Selon ce modèle, le comportement d'une personne serait déterminé par son intention comportementale à l'adopter. Cette intention serait quant à elle déterminée par l'attitude de la personne et par ses normes subjectives relatives au comportement en question ; étant donné que les auteurs, Fishbein et Ajzen, définissent les normes subjectives comme étant la perception de l'individu sur le fait que la plupart des personnes qui sont importantes à ses yeux, sont d'avis qu'il devrait ou ne devrait pas effectuer le comportement en question. Ainsi, selon la théorie de l'action raisonnée, l'attitude d'une personne envers un comportement serait déterminée par ses croyances envers les conséquences de ce comportement multiplié par son évaluation de ces conséquences. Rapportée à cette étude de la victimisation des employées mineures, les conséquences visées du comportement (qui n’est autre que l'acte victimel) sont un travail bien fait, un rendement efficace et acceptable, une servante ou mineure toujours à la tâche, prête à servir. Les croyances sont définies par la probabilité subjective de l'individu sur le fait qu'effectuer un comportement particulier va produire des résultats spécifiques comme l'employée mineure idéale que l'employeur se représente. L'intention d'effectuer un comportement est également déterminée par les normes subjectives qui sont elles-mêmes déterminées par les croyances normatives d'un individu et par sa motivation à se plier aux normes. La théorie de l'action raisonnée postule que les facteurs influençant le comportement le font de manière indirecte en agissant sur l'attitude ou les normes subjectives.

La théorie de l'action planifiée de Ajzen (1985, 1991) est une extension de la théorie de l'action raisonnée. Elle part du principe que trois variables telles que l'attitude, les normes subjectives et la perception du contrôle influencent directement les intentions d'effectuer un comportement et cette intention influence à son tour, le comportement. La perception du contrôle sur le comportement se réfère aux ressources dont dispose l'individu, à ses propres capacités, aux opportunités disponibles ainsi qu’à la perception de l'importance d'arriver à accomplir les résultats. Dans cette perspective, la référence à l'étude de Taylor et Todd que fait Edu Tech Wiki (2011) est d'une importance capitale, puisque ces auteurs indiquent l'existence d'antécédents de l'attitude, des normes subjectives et de la perception du contrôle. Les résultats auxquels ces auteurs ont abouti démontrent que les éléments qui déterminent la variable attitude sont la perception de l'utilité, la perception de la facilité d'utilisation et la 
compatibilité. Finalement, les conditions facilitatrices, les facteurs déterminant la perception du contrôle sur le comportement de l'employeur se résument à l'interprétation subjective, le jugement de valeur des agissements de l'employée mineure. Elle est à son tour déterminé par l'attente d'une personne idéale dévouée au travail domestique que veut atteindre l'employeur. Cette perception subjective des actes tenant compte de la compréhension et de la représentation des faits, gestes et paroles des employées mineures par les employeures-patrons conduit à victimiser ces employées.

Ces études citées sont pertinentes. Elles mettent l'accent sur les pratiques sociales en ce qui concerne l'éducation et la socialisation des enfants. Elles évoquent également les facteurs culturels de la pratique discriminatoire dans la distribution des tâches domestiques, la pérennisation de ces pratiques ainsi que la compréhension des actes qui poussent les parents, enseignants et tout autre individu à victimiser les enfants. Cependant, elles ne désignent pas ces pratiquent comme motivant et déclenchant l'acte victimel des parents, des employeurs. La présente étude se propose alors d'interroger aux plans social, culturel et personnel les éléments motivationnels et déclenchant des actes victimels des employeures de mineures de ménages. Alors, quel jugement l'employeur a-t-il du travail et du comportement de la mineure qui puisse le conduire à la victimiser ? Autrement dit, sur quoi se base t-il pour victimiser la mineure ? Quels sont les éléments culturels déterminant l'employeur à victimiser la mineure ?

\section{Objectifs}

Cette étude a pour objectif de connaître (identifier, décrire et expliquer) les pratiques culturelles et personnelles motivant les actes victimels de l'employeur(e).

\section{Hypothèses}

$>\quad$ La socialisation par le châtiment pour inculquer les normes sociales aux mineures et obtenir un meilleur rendement de leur part explique les actes victimels des employeur(e)s

$>\quad$ L'interprétation subjective des faits, gestes et paroles des mineures par les employeur(e)s explique la victimisation des mineures

\section{Méthodologie}

\section{Sujets}

La population d'enquête de cette recherche est composée des parents et de personnes ayant déjà employé des mineures. Les parents et employeurs sont des deux sexes, au nombre de trente-neuf (39) dont dix (10) de sexe masculin et vingt-neuf (29) de sexe féminin, exerçant dans divers secteurs de la vie active. Notre choix s'est essentiellement porté sur la commune de 
Yopougon où l'on dénombre la présence d'employées mineures dans de nombreuses familles. Cette présence est perceptible et l'on y rencontre des cas de violences exercées sur les mineures employées dans les ménages. Cela ne traduit nullement pas que des cas de victimisation n'existent pas dans les autres communes de la ville d'Abidjan. Bien au contraire, seulement le cas de Yopougon nous a particulièrement interpelés. Bien que faisant référence quelques fois à des statistiques, nous sommes conscients que le nombre de personnes avec qui nous avons échangé n'est pas représentatif de la population abidjanaise. Nous faisons une étude qualitative.

\section{Méthode}

La technique de recueil des données pour cette recherche est essentiellement l'entretien, notamment l'entretien semi-directif ; non seulement pour laisser les répondants s'exprimer librement, mais aussi de recentrer les questions sur les objectifs de l'étude. L'identification et l'accès à ces personnes étaient rendus difficiles. La technique de la boule de neige nous a été d'un précieux apport. En effet, la rencontre de quelques filles victimes et des personnes voisines ayant connaissance d'enfants domestiques violentées nous ont permis de rencontrer effectivement les trente-neuf personnes avec qui, nous nous sommes entretenues au cours de l'étude. Ces personnes étaient réticentes pensant que nous étions des envoyés d'un ministère et subiraient des représailles. Les échanges avec les parents et les employeurs abordaient la représentation de la violence sur les enfants dans le cadre de l'éducation familiale et les raisons favorisant les violences sur ces enfants. La méthode quantitative a permis de faire le traitement statistique des données recueillies. La méthode qualitative a servi à faire ressortir le sens des discours des personnes rencontrées.

\section{Résultats}

Les résultats de l'enquête sont structurés sur deux axes : socialisation par le châtiment et l'interprétation subjective des faits, gestes et paroles des mineures par l'employeure.

\section{Socialisation par le châtiment}

\section{Composantes de la socialisation et représentation sociale des châtiments} dans l'éducation des mineurs

Dès le jeune âge, les enfants apprennent à connaître les noms des objets et les parents leur demandent d'envoyer et de rapporter des messages à certaines personnes de la famille et de l'environnement immédiat. Ces enfants participent quotidiennement aux travaux de la maison par de petites tâches dont la complexité et la charge de travail augmentent progressivement au cours de leur développement. Dans l'esprit de l'Africain, un enfant ne 
peut apprendre et surtout bien apprendre, assimiler une conduite qu'en contact régulier avec ce que l’on veut lui montrer. Les parents pensent alors enseigner aux enfants les compétences pratiques et sociales nécessaires à la vie adulte. A quel âge l'enfant commence-t-il à participer aux travaux de la maison? Voici les réponses recueillies des répondants.

Tableau $n^{\circ} 1$ : Début de la participation aux travaux de la maison

\begin{tabular}{|c|c|c|c|c|c|c|c|}
\hline $\begin{array}{c}\text { Âge du début de } \\
\text { la participation } \\
\text { aux travaux de la } \\
\text { maison }\end{array}$ & 5 ans & 6 ans & 7 ans & 8 ans & 9 ans & 10 ans & 12 ans \\
\hline $\begin{array}{c}\text { Nombre de } \\
\text { personnes } \\
\text { répondant }\end{array}$ & 6 & 5 & 8 & 9 & 1 & 7 & 3 \\
\hline
\end{tabular}

Des 39 parents et personnes employant des mineures que nous avons rencontrés et avec qui nous avons échangés, neuf personnes situent l’âge du début de la participation de l'enfant aux travaux de la maison, à l'âge de huit ans. Huit personnes situent cet âge à sept ans. Pour sept autres personnes, c'est à dix ans que l'enfant participe aux travaux. Six autres pensent que c'est à cinq ans. Cinq parents situent cet âge du début à six ans. Trois autres pensent que c'est à douze ans et une personne parle de neuf ans. Au total, 17 personnes sur les 39 indiquent que les enfants participent aux travaux de la maison à l'âge de 7-8 ans. 11 autres indiquent 5-6 ans. 8 autres parlent de 910 ans et 3 personnes pensent que c'est à 12 ans qu'un enfant doit commencer à participer aux travaux domestiques. 28 parents et employeurs de mineures affirment que les enfants de 5-8 ans participent aux travaux domestiques. A la question qui cherchait à connaître les tâches confiées à l'enfant à ces âges indiqués ? Les réponses sont consignées dans le tableau $n^{\circ} 2$.

Tableau n² 2 : Tâches confiées à l'enfant (réponses multiples)

\begin{tabular}{|c|c|}
\hline Les travaux exécutés par l'enfant & Nombre de réponses \\
\hline Faire la vaisselle & 27 \\
\hline $\begin{array}{c}\text { Entretien de la maison ; balayer, essuyer } \\
\text { Faire la lessive, se rendre propre ; se laver, laver ses habits, } \\
\text { ranger ses affaires, dresser son lit... }\end{array}$ & 23 \\
\hline $\begin{array}{c}\text { Faire les petites commissions ; aller à la boutique, rapporter un } \\
\text { objet à quelqu'un... }\end{array}$ & 14 \\
\hline $\begin{array}{c}\text { Apprendre un métier ; cordonnerie, ferronnerie, vente d'eau et } \\
\text { de jus... }\end{array}$ & 6 \\
\hline Faire la cuisine & 5 \\
\hline
\end{tabular}

27 réponses affirment que l'on peut demander à l'enfant de faire la vaisselle. 23 autres optent pour l'entretien de la maison qui consiste à balayer et à essuyer la maison (sol et meubles). 22 réponses estiment qu'il faut plutôt demander à l'enfant de faire sa lessive, de se rendre propre, de ranger ses 
affaires, dresser son lit etc. 14 réponses soutiennent que l'enfant peut faire les petites commissions ; aller acheter quelque chose à la boutique, rapporter un objet à quelqu'un ou à un endroit etc. Pour 6 réponses, l'enfant peut apprendre un métier (cordonnerie, ferronnerie, menuiserie, la vente d'eau glacée et de jus de fruits...). Enfin, pour 5 réponses, l’enfant et surtout la jeune fille peut commencer à apprendre à cuisiner, non pas à préparer le repas de la maison, mais à cuire le riz, faire de la bouillie etc. Quels objectifs vise-t-on en confiant certaines tâches, même si la charge selon les parents est adaptée à leur âge, aux enfants à bas âge ? Les différentes raisons évoquées peuvent être regroupées en quatre séries de réponses.

Tableau $n^{\circ} 3$ : Facteurs explicatifs de la participation aux travaux à bas âge

\begin{tabular}{|c|}
\hline Facteurs explicatifs de la participation \\
\hline 1.Donner le goût du travail à l'enfant \\
\hline 2.L'âge idéal de l'apprentissage pour l'enfant \\
\hline 3.Mieux apprendre, bien connaître le travail \\
\hline 4.J'ai commencé à travailler à cet âge (transmission intergénérationnelle) \\
\hline
\end{tabular}

Les parents et employeurs soutiennent qu'il faut donner le goût du travail à l'enfant qui devra grandir avec. Ensuite, il s'agit pour les personnes rencontrées, de l’âge idéal de l'apprentissage pour l'enfant, d'où celui-ci peut commencer à apprendre pour bien connaître la tâche qu'il accomplit et enfin, les parents et employeurs rencontrés affirment avoir commencé à travailler à cet âge aussi bas. Ils ne veulent pas laisser leurs enfants grandir sans rien apprendre surtout pour les différentes surprises que nous réserve la vie, l’on ne sait comment et où l'enfant passera le reste de sa vie. Il faut alors lui apprendre à affronter seul les difficultés quelque soit l'endroit où il pourrait se retrouver. A ce propos, voici quelques témoignages de parents avec qui nous avons échangé au cours de l'étude. Pour un sans emploi de 27 ans de niveau d'étude secondaire, "mon enfant participe aux travaux de la maison à l'âge de 10 ans. Il entretient la maison en balayant et en essuyant. Il fait la lessive et prépare la nourriture pour mieux apprendre et être courageux. Ce qui l'amènera demain à être une bonne mère de famille ». Pour un infirmier d'Etat âgé de 50 ans, "mon enfant commence à participer aux travaux de la maison à l'âge de huit ans. Il fait tout ce que les enfants doivent apprendre c'est-à-dire à faire la lessive, la vaisselle et autres. Car on ne sait pas ce que l'avenir nous réserve. Je peux être absent; en cas de décès par exemple, c'est pourquoi je les forme dès maintenant ». Pour un auditeur et contrôleur de gestion de niveau d'étude supérieure âgé de 55 ans, " c'est à partir de 6-7 ans que mon enfant commence à participer aux travaux de la maison. Il s'agit des petites commissions; apporte ceci, ramène cela. Cet âge correspond à l'âge idéal de l'apprentissage. Pour moi, il faut faire comprendre à l'enfant qu'il faut nécessairement travailler dans la vie ». 
Selon une ménagère de niveau d’étude correspondant au premier cycle du secondaire, âgée de 49 ans, « déjà à partir de 5 ans, mon enfant commence à participer aux travaux de la maison. Je lui apprends à laver ses propres vêtements. Il apprend à faire la vaisselle et à nettoyer la maison. Il commence aussi tôt pour qu'il soit habitué et grandir avec. Il n'est pas évident que l'enfant passe toute son enfance et son adolescence à mes côtés ». Une commerçante de niveau secondaire, âgée de 40 ans soutient «dès le bas âge, c'est-à-dire à partir de 7-8 ans, les enfants de sexe masculin commencent à laver leurs habits. Les filles, en plus de la lessive, apprennent à préparer afin que ces enfants s'habituent, assimilent vite et bien. A cet âge, les enfants s'adaptent mieux et obéissent. Tout simplement, le travail est formateur et c'est à bas âge qu'il faut former le caractère, la rigueur de l'enfant ». Une institutrice de niveau d'étude supérieur âgée de 26 ans avance qu' "au minimum, l'enfant doit commencer à participer aux travaux de la maison à partir de l'âge de 10 ans. L'on peut lui confier les petits travaux de la maison; la vaisselle et sa propre lessive le jour il ne va pas à l'école, puisqu'on ne sait pas ce que la vie nous réserve. Demain par exemple, il peut se retrouver chez une tierce personne, un tuteur ou autre et il devra réussir à s'en sortir seul en donnant une bonne image de sa famille d'origine. Mes sœurs, mes frères et moi-même avons appris à représenter dignement notre famille partout où nous passons et les gens viennent le témoigner à nos parents. Nous devons et avons le devoir d'apprendre cela à nos enfants ». Pour une couturière et une caissière de niveau d'étude secondaire, âgées respectivement de 40 ans et 39 ans les points de vue ne diffèrent pas. Selon elles, "l'âge de sept ans est idéal pour que l'enfant commence à participer aux travaux de la maison. L'on peut lui demander de balayer et essuyer la maison. On peut également lui apprendre à faire sa propre lessive pour ne pas qu'il soit paresseux puisqu'à un certain âge, l'enfant doit apprendre à aimer le travail. Il doit s'habituer au travail, affronter la vie seul, car il n'est pas sûr et certain qu'il poursuive son développement à nos côtés...». Tels sont les résumés des réponses à l'interrogation cherchant à connaître les raisons et l'âge du début de la participation de l'enfant aux travaux de la maison.

Quels sont les moyens utilisés pour donner ce goût du travail à l'enfant ? Le terme '’moyens' s'entend toutes les stratégies de transmission théorique uniquement ou associée à la pratique et la transmission théorique associée à la pratique plus la sanction pour recentrer, corriger les déviations de l'enfant-apprenti. 25 réponses indiquent que pour donner le goût du travail à l'enfant, il faut associer les paroles à la pratique. "Pour montrer mes connaissances à mes enfants, je le fais de façon théorique, par les paroles et les exemples que je leur donne en exécutant la tâche en leur présence ». 15 réponses soutiennent qu'il faut impliquer l'enfant au cours de 
la transmission des connaissances. "Pour montrer mes connaissances aux enfants, je le fais théoriquement. Ensuite, j'implique l'enfant et je veille à ce qu'il exécute la tâche en ma présence ». 9 réponses montrent qu’il faut transmettre les connaissances uniquement par la théorie, par les paroles. La sanction doit-elle être associée à la transmission au cours de l'apprentissage? Les 39 personnes répondent en affirmant qu’il est nécessaire d'associer la sanction à la transmission pour que l'enfant prenne conscience et voit l'importance de ce qu'on lui montre. Quelle sanction estelle nécessaire pour réussir une socialisation admise par tous ? 29 réponses souhaitent les châtiments corporels, les frappes, les fessées. 11 réponses sont favorables aux injures dévalorisantes et humiliantes. 7 réponses optent pour les menaces fermes qui montrent le sérieux de ce qu’il apprend. 4 réponses projettent les négligences et les isolements, l'ignorance de l'enfant.

Pour l'Africain donc, très tôt, à bas âge, aux environs de 5-6-7-8 ans, l'enfant doit apprendre l'amour du travail en participant aux travaux de la maison. Cet apprentissage doit être un ensemble de théories, de paroles donnant les directives suivies de pratiques, d'exemples associant ou impliquant l'enfant et des sanctions positives ou négatives. Généralement, les châtiments corporels, les fessées et autres coups et frappes de socialisation sont plus que nécessaires pour inculquer les connaissances de morale, de savoir vivre et de savoir faire. A la question de savoir comment les répondants qualifient leur méthode de transmission de valeurs et croyances aux enfants? Les 39 personnes rencontrées pensent que leur méthode de socialisation est bonne. «Pour moi, les moyens de socialisation utilisés sont bons. La rigueur, les châtiments dans l'éducation sont vraiment importants. Ils permettent de faire peur aux enfants et par conséquent, ceuxci respectent les interdits ». "Mes moyens de socialisation sont acceptables dans la mesure où les menaces que j'adresse à mes filles font qu'elles m'écoutent, suivent mes conseils et font bien ce que je leur demande. Pour le moment, j’ai moins de soucis avec elles». "Mes moyens utilisés sont acceptables. Les injures dévalorisantes et humiliantes ainsi que les négligences et les isolements font prendre conscience aux enfants qui ne supportent pas les injures. Pour les éviter, ils font bien ce qu'ils ont à faire. Tous mes enfants qui sont allés vivre ailleurs me font honneur puisqu'ils sont très bien acceptés dans les familles d'accueil et celles-ci ne font que nous remercier chaque fois qu'elles en ont l'occasion". "Les châtiments que j'utilise actuellement comme moyens de socialisation sont très bons, puisqu'autre fois, j'utilisais les menaces et les injures dévalorisantes et humiliantes mais j'ai constaté que mes enfants ne m'écoutaient pas et étaient presque gâtés, pourris même. Le fait d'être très sévère et rigoureux permet aux enfants de se redresser et suivre les consignes des parents. Ce sont des stratégies dissuasives et elles marchent très bien avec mes enfants ». 
Au total, les personnes rencontrées bien que non représentatives de la population Abidjanaise, sont favorables aux châtiments corporels, aux injures dévalorisantes et humiliantes, aux menaces, aux négligences et aux isolements comme sanctions pour l'adoption des comportements interdits et le non respect des consignes parentales au cours de la socialisation, de la transmission des connaissances, des valeurs et croyances culturelles aux enfants. Au travers des informations recueillies et à notre possession, nous pouvons soutenir que les africains utilisent traditionnellement la violence, même d’intensité modérée, au cours de la socialisation des enfants. Qu'est ce qui explique donc un tel comportement ? Un aspect non moins important qui encourage et favorise inconsciemment cette tendance culturelle de l'utilisation de la violence au cours de la socialisation est la caution implicite de la société qui reste de marbre, muette face à la victimisation d'une mineure.

\section{Consentement tacite ou caution implicite de la société}

A la question, le voisinage intervient-il lorsque vous frappez votre enfant ? La majorité des personnes rencontrées donne une réponse négative. En effet, trente (30), (77 \%) des répondants affirment que personne du voisinage n'intervient au cours des bastonnades portées à un enfant. D'ailleurs, soutiennent-ils «c'est une affaire privée. La correction de mon enfant ne concerne que moi seul. Ce qui se passe dans ma maison est mon affaire et non une affaire de tous ». "Avant, au village, l'éducation de l'enfant était une affaire publique. Lorsqu'un enfant d'une tierce personne avait un mauvais comportement, une personne $X$ pouvait lui porter des coups de correction socialisante sans problème avec les parents géniteurs. Un enfant ainsi que son éducation appartenaient à tout le monde. Aujourd'hui, ce temps là est révolu. Personne n'a le droit de toucher, de châtier son propre enfant à plus forte raison, l'enfant de quelqu'un d'autre ». "Vous savez, punir, corriger un enfant est une bonne chose. Tant que vous demeurez au niveau des fessées de redressement, vous êtes dans vos droits parentaux de socialisation. Mais les châtiments sévères très fréquents attirent l'attention du voisinage, au point où certains voisins viennent vous donner des leçons chez vous dans votre maison ». "Corriger un enfant est normal si vous ne voulez pas que l'enfant soit 'gâté' quand il sera adolescent ou même adulte. Cependant, je ne veux pas me mêler de ce qui ne m'intéresse pas. C'est de cette même manière que je veux être tranquille lorsque j'ai des problèmes avec ma famille ». Pour les neuf autres personnes, soit 33 \% de l'échantillon, des individus ne faisant pas partie de la famille interviennent souvent pour demander pardon, bien qu'ils reconnaissent le bien fondé de la punition ou de la correction parentale. Résumons les discours que ceux-ci tiennent : «Tous les enfants de nos jours 
sont ainsi. Il faut vous maîtriser sinon la colère est mauvaise conseillère. Un jour, dans votre colère, vous allez commettre le pire sans vous en rendre compte. Allez-y doucement avec eux...». Nous remarquons que les répondants et les personnes dont les discours sont rapportés sont favorables aux fessées, aux corrections mais laissent la latitude à chaque parent d'éduquer son enfant de la meilleure manière qu'il soit. Cette tendance culturelle influe-t-elle sur les pratiques personnelles de la victimisation des enfants?

\section{Interprétation subjective des actes des enfants}

Les facteurs explicatifs de la victimisation des enfants dans leur famille tiennent également aux facteurs personnels du parent ou du victimiseur. En effet, la victimisation d'un enfant est fonction de la compréhension des actes, gestes, paroles de ce dernier par le parent. Cette compréhension dériverait de la personnalité de chaque individu. Or cette singularité individuelle aurait une base non seulement du bagage inné, mais également sociale, culturelle. L'individu a son propre caractère, sa propre manière de voir, de comprendre les choses et de les rendre dans les relations aux autres. Ce caractère a certainement des référents sociologiques, car l'individu, bien qu'ayant son caractère propre à lui, fait très souvent référence aux tendances culturelles de socialisation dont il a lui-même bénéficiées. A la question d'indiquer les actes concrets de l'enfant qui pourraient susciter la réaction de victimisation du répondant, les réponses à cette interrogation exposent le refus de travailler de l'enfant. Un enfant paresseux qui ne veut rien faire dans la vie, qui exécute mal les tâches que l'on lui confie. "Les actes concrets de l'enfant qui m'amènent très souvent à utiliser les menaces, c'est quand je ne suis pas satisfaite du travail qu'il a fait ». "Quand je confie une tâche à l'enfant et qu'il n'arrive pas à me satisfaire, j'utilise des châtiments corporels pour l'amener à comprendre qu'il doit mettre tout le sérieux dans ce qu’il exécute ». "Si l'enfant est distrait, refuse de faire des efforts pour assimiler ce que je lui montre, je l'insulte bien, même si c'est devant les autres membres de la famille ». "Les actes de l'enfant qui peuvent m'amener à le frapper, c'est quand l'enfant n'est pas attentif, il ne s'intéresse pas à ce que l'on lui montre et très souvent exécute maladroitement les tâches que je lui confie ». L'enfant est paresseux, fainéant, distrait, n'est pas attentif, ne veut rien faire dans la vie, ne me donne pas satisfaction etc. relève de la subjectivité car toutes ces appréciations dépendent de la compréhension de chacun. Les efforts fournis par l'enfant peuvent être compris autrement par une autre personne. C'est pourquoi nous avons voulu savoir si les parents ou employeurs de mineures sont conscients de leurs agissements avec les enfants. Comment qualifiezvous vos attitudes et comportements avec vos enfants? " J'ai un 
comportement dur envers mes enfants". "Pour amener mes enfants à intérioriser ce que je leur montre, j'utilise les châtiments corporels et les menaces et je trouve que c'est très sévère de ma part. Cependant, je pense que les enfants ont besoin de cela pour obéir et exécuter ce qu'on leur demande ». "Pour faire assimiler un savoir faire aux enfants, les moyens utilisés sont les châtiments et les négligences ou isolements. Je trouve que cela n'est pas bon, mais je les utilise quand même car les êtres humains et surtout les enfants ont besoin de la fermeté, de la rigueur donc de la violence pour faire ce qu'on leur demande. Je trouve que cela est bien pour cet âge et les enfants têtus afin qu'ils ne grandissent pas avec ce mauvais caractère ». «Pour faire assimiler mes connaissances à mes enfants, j'exécute le fait à leur présence et leur demande de le reprendre. Au début, j'utilise les menaces quand la tâche est mal faite. En fin de compte, lorsque je me rends compte que l'exécution et l'assimilation sont quasi impossibles, j'emploie les moyens forts, c'est-à-dire les châtiments. J'essaie donc de les comprendre, mais je suis tout de même rigoureux et ferme dans les relations avec les enfants. Ils ont besoin d'un tel équilibre entre la manière forte et la douceur ». "Les châtiments sont très bien pour l'éducation des enfants. Car tout compte fait, tout ce que nous montrons est notre manière de les éduquer et avec ces moyens, les enfants apprennent mieux. Moi je pense que c'est bon pour eux ». "J'ai un comportement très dur avec mes enfants. Je trouve qu'il faut souvent cela pour montrer aux enfants que la vie n'est pas aussi facile qu'ils le pensent». "Je pense avoir une attitude acceptable avec les châtiments pour les mettre sur le chemin de la socialisation. Mais mes enfants disent que souvent je suis très sévère avec eux. Pourtant, moi je trouve que souvent, il faut la violence et la fermeté pour montrer aux enfants qu'ils sont sur la mauvaise voie, une vie inacceptable». "J'utilise les injures dévalorisantes, humiliantes et autres ainsi que les négligences ou les isolements pour signifier à l'enfant que ce qu'il fait n'est pas bon. Parfois, je suis attentive mais très souvent sévère afin que ceux-ci sachent les deux facettes du comportement humain et ainsi soient préparés psychologiquement ».

En fin de compte, les personnes rencontrées sont conscientes des réactions violentes qu'elles adoptent face aux agissements des enfants. Elles réalisent même qu'il s'agit de la meilleure méthode d'éducation, de socialisation puisque les enfants ne sont pas concentrés, n’ont pas conscience de ce qu'ils apprennent et comme la tortue, les mineurs ont besoin d'être boostés, qu'il faut pour ces raison les canaliser, les remettre sur les rails en employant la manière forte. Qu'est-ce qui pourrait motiver un tel raisonnement ? Les parents et employeurs de mineures ont fait référence à leur culture d'appartenance pour nous confier les réponses que nous résumons ici. «Dans ma culture, l’on fait référence à la violence pour la 
réussite de l'enfant. Vous voyez, moi-même je suis passé par là. J'ai été éduqué dans ces conditions pour m'empêcher de poser les actes déviants et bien exécuter les tâches que les parents me confiaient. Ma culture accepte la violence pour sa force dissuasive ». "Selon notre culture, la violence au cours de la socialisation a une force éducative. Les fessées, les injures permettent aux parents d'être respectés et suivis par les enfants. Dans cette perspective les enfants qui veulent éviter les humiliations et la honte se mettent au pas de tout ce que les parents entreprennent ». "La punition par les châtiments sévères n'est pas une bonne méthode. Il faut des punitions modérées, selon ma manière de comprendre la vie. Sinon, dans ma culture, l'on trouve en la violence un pouvoir éducatif. C'est un moyen de communication très important qui ramène l'enfant à l'ordre. C'est donc un moyen de dépréciation, du rejet des mauvais agissements d'un enfant». «Ma culture n'interdit pas la correction des enfants, seulement trop de violence tue l'éducation. La plupart des enfants sont turbulents, distraits et immatures. Leurs comportements sont très souvent indésirables. C'est en ce sens que la violence est acceptée dans ma culture pour redresser et dresser l'enfant. Il est alors important de frapper l'enfant quelque fois lorsque ce dernier n'obéit pas aux parents ». "Chez nous en Côte d'Ivoire, et particulièrement au nord, la violence est admise dans l'éducation pour faire prendre conscience des interdits sociaux à l'enfant, car l'adoption de tels comportements entraîne des sanctions très sévères. Mes enfants par exemple quand ils sont têtus, je réagis vigoureusement et ils changent d'attitudes sur le champ. Ils savent que je n'aime pas qu'ils déshonorent la famille avec de tels comportements rejetés par tous ».

Ainsi, dans la culture africaine et particulièrement celle de la côte d'Ivoire, les fessées, les frappes et autres menaces et injures de socialisation sont admises et acceptées pour leur force dissuasive et éducative. Cette conclusion nous amène à nous interroger sur la nécessité de la violence dans toute éducation, tout apprentissage. A cette question de la nécessité du besoin de la violence dans toute éducation ou tout apprentissage, les parents et employeurs donnent les réponses regroupées dans le tableau $n^{\circ} 4$ suivant.

Tableau $\mathrm{n}^{\circ} 4$ : Besoin de violence dans toute éducation

\begin{tabular}{|c|c|c|c|}
\hline Nécessité de la violence dans toute éducation & Oui & Non & Total \\
\hline Homme & 4 & 6 & 10 \\
\hline Femme & 13 & 16 & 29 \\
\hline Total & 17 & 22 & 39 \\
\hline Pourcentage (\%) & 44 & 56 & 100 \\
\hline
\end{tabular}

Quatre hommes et treize femmes, soit $44 \%$ répondent par l'affirmative alors que six hommes et seize femmes, soit $56 \%$ disent non. L'on n'a pas forcement besoin de violence dans toute éducation. Six des femmes qui répondent non, nuancent leur propos. «Toute éducation n’a pas 
forcement besoin de violence, mais il existe des situations où la menace et autres mesures dissuasives peuvent faire peur et l'enfant peut obéir et très bien exécuter la tâche qu'on lui confie ». "Quelques fois, non mais par moment, il faut cela. Pas forcement la violence mais il faut cela souvent », etc. Si l'on ajoute donc ces six femmes aux dix-sept autres qui expriment ouvertement sans ambigüité la nécessité de la violence dans toute éducation, nous obtenons vingt-et trois (23) sur les 39 soit 59 \% des répondants qui sont favorables à la nécessité de la violence dans toute éducation. Cette tendance à recourir à la violence au cours de la socialisation intériorisée par chaque individu influence-t-elle la qualification des agissements et le passage à l'acte victimel ? Selon les personnes rencontrées, répondre par l'affirmative à cette question, c'est rechercher la goutte d'eau qui ferait déborder le vase. C'est-à-dire qualifier négativement les agissements des mineures pour pouvoir les corriger, les frapper. C'est justifier en quelque sorte l'acte victimel que le parent ou l'employeur veut poser. Une telle compréhension explique la majorité du non sur le oui. En effet, les trente-neuf personnes disent qu'il n'existe pas de lien entre la tendance à faire recours à la violence et la qualification des actes de l'enfant. L'appréciation des agissements de l'enfant est alors fonction de la compréhension de chaque parent ou employeur et du comportement concret de l'enfant. "Il y a des enfants qui ne respectent pas les règles de vie en société malgré tous les efforts fournis par les parents pour leur donner une bonne éducation. Une telle qualification n'a aucun lien avec le fait que je veuille leur porter main. Seulement, le comportement étant mauvais, je peux le frapper en voulant le corriger. Le contraire qui voudrait que je n'accepte pas ou rejette ses actes pour le chicoter n'est pas juste ». "Certains enfants sont paresseux et n'ont aucune envie de faire quoique ce soit. Les travaux qu'on leur confie sont mal exécutés. Je ne suis donc pas toujours content de leurs agissements. Quelques uns donnent des satisfactions qui sont les fruits des corrections, des insistances pour apprendre ce qu'on leur montre. C'est donc le travail et le comportement de chaque enfant que nous qualifions et non notre volonté de les frapper qui amène à de faux jugements ». "Quand je dis que les enfants sont respectueux, ce sont les actes que je qualifie. Lorsque je dis aussi qu'il sont impolis, irrespectueux, insupportables etc. c'est également leurs comportements que j'apprécie. Je n'ai aucune intention de les frapper, mais je soutiens qu'il faut la chicote pour redresser les enfants insupportables. J'aime quand mes enfants respectent et font ce qu'il faut en ma présence comme à mon absence ». "Les enfants sont non seulement des paresseux, mais ils sont irrespectueux. Il faut leur apprendre le travail ainsi que comment respecter. Quand je leur confie des tâches, ils ne les font pas correctement. Ils aiment la facilité et apprennent à bouder les grandes personnes, à les regarder avec mépris, et tout cela c'est du banditisme. En 
tout cas, ils ne sont pas des exemples à suivre. C'est ce qui m'oblige à utiliser la chicote pour leur exprimer mes désaveux». "Je qualifie leurs comportements d'indésirables puisqu'ils ne sont pas exemplaires. Ils traduisent bien l'éducation qu'ils ont reçue à bas âge chez leurs parents qui ont voulu suivre, je dirais les mauvais conseils des autres en ne corrigeant pas les fautes des enfants et ceux-ci croient être sur le bon chemin. Aujourd'hui, il est difficile de rattraper ces erreurs. Les enfants sont gâtés, et moi, puisqu'ils ne sont pas mes vrais enfants, je suis obligée de faire un peu attention. Mes propres enfants n'aimaient pas le travail, mais actuellement, ils sont presque parfaits, des exemples quand ils se retrouvent ailleurs ». «Mes enfants ne m'obéissaient pas quand je leur parlais. Ils me fatiguaient et je les aimais malgré ces défauts car j'ai besoin d'eux. Je me suis dit les élèves sont devenus nuls parce que dit-on, les maîtres ne doivent plus frapper les élèves. Ils n'ont plus peur de rien, c'est à peine même qu'ils écoutent leur maître. J'ai réfléchi et la résolution est qu'il me fallait les redresser. En tout cas, le travail que j'ai réalisé en un temps record les a amenés à être tous droits et présentement, ils deviennent des exemples. C'est donc le comportement des enfants qui fait changer. L'amour pour eux m'a poussé à faire d'eux des hommes de demain ».

Pour finir, la qualification des actes des enfants n’obéit pas à une logique de leur victimisation, comme le diraient les sages, que quand le lièvre veut manger le levraut, son petit, il prétexte que ce dernier sent le bouc. Les répondants se disent très loin d'un tel raisonnement et mettent à l'écart une démonstration pareille. La qualification des actes des enfants procède selon eux, d'une observation et de la description de leurs agissements se basant sur un ensemble de critères acceptés par les membres de la société. Les enfants de nature sont insupportables de par leurs comportements. Il appartient aux parents de les canaliser, de les orienter pour intérioriser les normes sociales.

Après analyse, nous remarquons l'existence de référents subjectifs bien que les répondants soutiennent se baser sur des critères objectifs pour qualifier les actes, paroles et gestes des mineurs. Car les critères d'appréciation de la paresse, de l'enfant fainéant, qui ne donne pas satisfaction, qui ne veut rien faire dans la vie etc. relèvent de la compréhension subjective de la personne vivant avec l'enfant et qui apprécie ses agissements.

\section{Discussion et conclusion}

Cette étude réalisée sur trente-neuf parents et employeurs de mineures a pour objectifs de connaître les pratiques socioculturelles et personnelles motivant les actes victimels du parent et/ou de l'employeur. Se basant sur l'hypothèse de recherche affirmant que la socialisation par le 
châtiment pour inculquer les normes sociales aux mineurs et obtenir d'eux un meilleur rendement, une obéissance, le respect des parents, des ainés et autres personnes, se trouve ici confirmée par les réponses des personnes rencontrées au cours de l'étude. Elles montrent que traditionnellement et culturellement, les ivoiriens et partant les africains utilisent la violence, même d'intensité modérée, au cours de la socialisation des enfants. Salem (2010) l'a également souligné en avançant que les parents et la société entière sont clairement favorables à la fessée comme méthode éducative. Ojeda et al. (Gautier 2004) évoquent le terme de nécessité et vont au-delà de l'acceptation de la fessée en soutenant que culturellement, dans les différentes sociétés africaines, le châtiment physique est nécessaire pour l'éducation des enfants. La seconde hypothèse soutient que la victimisation des mineures par les employeurs s'explique par leurs interprétations subjectives des actes, gestes et paroles de ces mineures. Les employeurs et parents rencontrés affirment se baser sur des critères objectifs pour la qualification des agissements des mineurs. Cette objectivité est certes mise en évidence par l'existence de référents socioculturels tels que le soutient et le confirme la première hypothèse, mais l'existence également d'aspects psychologiques pour la compréhension du comportement des mineurs est indéniable, puisque tous les individus de la même origine socioculturelle n’ont pas tous les mêmes appréciations et tous ne victimisent pas les mineurs qu'ils emploient dans leurs différents ménages. La victimisation des mineures employées de ménage à Abidjan est alors fonction du jugement de valeur de l'employeur. Okpo (2010) indiquait que l'interprétation subjective des actes des enfants et adolescents était déterminante pour la victimisation de ceux-ci, et que la faute inhérente à l'enfant victime pouvait être minimisée, même s’il fallait en tenir compte dans le processus de victimisation, puisque ce comportement déclenchant la victimisation de la mineure n'est pas compris de la même façon par tous. Certaines personnes pensent même que ces actes ne peuvent pas emmener un éducateur à frapper l'enfant que l'on devait laisser développer ses capacités physiques et intellectuelles même s'il faut de temps à autre le canaliser.

En fin de compte, les déterminants psycho-socioculturels de la victimisation des mineures se mesurent en trois éléments essentiels. L'interprétation subjective des agissements de la mineure, c'est-à-dire le jugement de valeur du comportement de la mineure par l'employeurvictimiseur. Ensuite, l'acceptation des châtiments corporels en Afrique et particulièrement en Côte d'Ivoire pour leur force dissuasive et éducative. Taylor et Todd (Edu Tech Wiki, 2011) avaient déjà indiqué que celui qui porte des coups synonymes de correction de socialisation à un mineur dans les sociétés africaines ne commettait pas de faute au plan social. Enfin, la conscience que ce fait restera impuni et l'intériorisation de cette violence 
admise par l'ensemble du corps social qui donne tacitement sa caution en ne réagissant pas, encourage les employeurs à porter des coups, à exercer des violences, à victimiser tout simplement la mineure, fort de son impunité acceptée de tous. Evoquant l'impunité et la caution tacite de la société, (sans le mentionner explicitement), Gautier (2004) avance que des auteurs indiens indiquent l'existence de violences subies par les enfants au sein des familles, surtout la notion de "'parternalisme'” permet de comprendre le rôle social de ces violences pour '’l'éducation'” des enfants. L'auteure ajoute même que de nombreuses sociétés pratiquent ou ont pratiqué les châtiments corporels pour inculquer aux enfants les normes sociales en vigueur. Wyss (2006) indique également le rôle déterminant que jouent la société entière et les chercheurs. Car écrit-elle, l'étude de la violence féminine n’était pas jugée prioritaire, probablement parce qu'elle est aussi dérangeante que la violence des femmes contre les hommes et les rapports sur la violence domestique mentionnent la nécessité de protéger les enfants contre la violence du père, mais pas de la mère... La mère peut alors victimiser une mineure sans crainte. Pour l'UNICEF (2000), bien que les rapports de force historiquement inégaux entre hommes et femmes soient les causes de la violence domestique, la peur et le contrôle de la sexualité féminine, la conviction de la supériorité inhérente de l'homme et les diktats législatifs et culturels qui ont traditionnellement refusé aux femmes et aux enfants un statut juridique et social expliquent et représentent les facteurs de perpétuation de la violence domestique. Salem (2010) soutient une telle thèse en avançant l'existence de mécanismes transgénérationnels associés à la maltraitance. Selon lui, la perspective existentielle et éthique de l'approche contextuelle de Boszormenyi-Nagy met en exergue le mécanisme généralisé de la légitimité destructive, sorte de 'bon droit' à faire le mal, qui peut se perpétuer dans une famille de génération en génération. Nos objectifs visaient à identifier, à décrire et à comprendre les déterminants socioculturels et personnels à l'origine de l'adoption de conduites tendant à victimiser les mineures. Le recours à la théorie culturaliste associée aux théories de l'action raisonnée et de l'action planifiée a abouti à la connaissance des motivations des conduites victimelles des employeurs. Sans une grande marge d'erreurs, nous pouvons avancer que nos objectifs ont été atteints et que les hypothèses de recherche ont été confirmées d'autant plus que les châtiments corporels et les violences psychologiques sont plus que nécessaires et les africains, partant les ivoiriens leur reconnaissent une force dissuasive et éducative. Aussi, les jugements de valeur déterminent-ils les employeurs à victimiser les mineures.

Au total, le mutisme coupable de la société (faut-il encore préciser traditionnelle), la conscience de la caution tacite quant à l'impunité de celui qui châtie un mineur, même s'il s'agit de son enfant, (donc de l'imprégnation de l'esprit), l'admission de la violence au cours de l'apprentissage ou de la 
socialisation du mineur et les jugements de valeur ou l'interprétation subjective, la perception des actes de l'enfant selon sa personnalité sont les principaux déterminants psycho-socioculturels de la victimisation des mineures employées de ménages à Abidjan. Cette victimisation est alors l'ensemble des stratégies des employeures pour obtenir des mineures un dévouement au travail, une disponibilité et autre rendement acceptable méritant le salaire mensuel des employées. De tels déterminants psychosocioculturels nous interpellent pour des réflexions sur le vécu et la perception de la victimisation par les employées mineures et les déterminants de toute victimisation. Car comme l'indique Mugnier (2010), ces explications ou idées perpétuées au cours des générations permettent de garder durablement le silence et tentent de faire comprendre que si le parent fait du mal, c'est pour le bien de l'enfant et les maltraitants laissent très souvent entendre, ce que nous faisons est mal, mais nous avons le droit de le faire puisque nous ne nous faisons pas de mal.

\section{References:}

1. Ajzen, I. (1985). From intention to actions: Atheory of planned behavior. In J. Kuhl \& J. Beckmann (Eds.), action control: From cognition to behavior (pp.11-39). Heidelberg: Springer

2. Ajzen, I. (1991). “The theory of planned behavior”. Organizational behavior and human decision processes, vol.50, pp.179-211

3. Coulibaly, M. L. (2010). Victimisations, climat et institutions scolaires: Essai de reconstruction du concept de violences scolaires comme objet d'étude à partir d'une comparaison Sénégal-France. Thèse de doctorat de $3^{\text {ème }}$ cycle en Sciences Humaines et Sociales. Ecole Doctorale des Sciences Sociales: Société, Santé, Décision. Mention Sciences de l'éducation. Thèse non publiée mais en ligne : file://E:/Coulibaly.pdf

4. Chaoui, M. (2009). Les “'petites bonnes”' ou enfants domestiques au Maroc (consulté le 9.5.2016). Site web file://E:/Les.htm

5. Edu Tech Wiki (2011). Théorie de l’action raisonnée. (consultée le 27-5-2016). Site web : « http:///edutechwiki.unige.ch/fmediawiki/index.php?title=The orie_de_l'action_raisonnée\&oldid=21433”

6. Gautier, A. (2004). Les violences au sein de la famille. Dans Quesnel A. (ed), Rapport des experts français à la 35e session Population et développement, Paris, Ministère de l'emploi et de la solidarité, Direction de la population et des migrations, CEPED.

7. Gilles, S. (2013). Enfance en danger, enfance maltraitée. Mieux cerner le phénomène pour mieux agir: le rôle de l’ONED. Dans 
Maltraitance et protection de l’enfance en France : état des lieux. Sénat. Paris

8. Jacquemin M. (2002). Travail domestique et travail des enfants, le cas d'Abidjan. Revue Tiers-Monde, t. XLIII, n¹70

9. Linares, J-L. (2008). Des abus et autres maltraitances: la maltraitance familiale, entre thérapie et contrôle. De Boeck. Carrefour des psychothérapies

10. Mejjati, A. R. (2002). Le travail des enfants au Maroc: approche socio-économique. Rapport sur le travail des enfants. Understanding children's work An Inter-Agency Research Cooperation Project. Rome

11. Moreno, M. (2015). Les “'petites bonnes'” du Maroc refusent de se taire (consulté le 9-5-2016). Site web: http://www.tdh.ch/fr/news/maroc-petites-bonnes-mais-grandesvictimes.

12. Mugnier, J-P. (2010). D’un silence partagé à un récit mis en commun: de la nécessité de bien pouvoir se raconteur. Cahiers critiques de thérapies familiales et de pratiques de réseaux $n^{\circ} 44$. De Boeck Supérieur. URL : http://www.cairn.info/revue-cahierscritiques-de-therapie-familiale-2010-1-page-79.htm

13. Okpo, N. A. (2010). Perception subjective des actes et victimisation des enfants et adolescents à Abidjan. Revue Internationale de Recherches et d’Etudes Pluridisciplinaires, n²17, pp.71-101. GUREP, Besanca, France

14. Raphaël, C. (2015) La démesure : soumise à la violence d'un père. Le livre de poche

15. Salem, G. (2010). Le droit de faire du mal aux siens. Cahiers critiques de thérapie familiale et de pratiques de réseaux $n^{\circ} 44$. De Boeck Supérieur

16. Salem, G. (2010). Maltraitance familiale: facteurs de risques transgénérationnels, in violence domestique: une interaction de facteurs de risque ? Actes du 5è forum violences domestiques, 18 mars 2010.

17. Schoebi, D. Plancherel, B. Tchumakov, m. Perrez, M. (2006). Aspects interculturels du fonctionnement relationnel et familial: la punition corporelle des enfants en Suisse et en Russie. Fontaine, Anne-Marie ; perrez, Meinrad - La revue internationale de l'éducation familiale, vol. 19, pp.53-75. Association internationale de formation et de Recherche en éducation familiale (AIFREF)

18. Tursz, A. \& Rousseau, D. (2013). Définition, fréquence et coût de la maltraitance. Idées reçues et faits démontrés. Dans Maltraitance et protection de l'enfance en France : état des lieux 
19. UNICEF (2000). La violence domestique à l'égard des femmes et des filles. Digest Innocenti $n^{\circ} 6$, centre de recherche Innocenti. Florence-Italie

20. Wyss, E. † (2006). Violence féminine : mythes et réalités. Quatrième rapport de la commission cantonale de l'égalité c/o Bureau cantonal de l'égalité, Berne 